\title{
Efecto de bioproductos en la producción de Phaseolus vulgaris L. y Arachis hipogea L.
}

\author{
Effect of bioproducts in the production of Phaseolus vulgaris L. and Arachis hipogea L.
}

\section{Tania Lambert G. ${ }^{1}$; Ramón Santiesteban S. ${ }^{2}$; Wilson Geobel Ceiro C. ${ }^{3}$; Manuel Enrique Fernández P. ${ }^{4}$; Guadalupe de las Mercedes López C. ${ }^{5}$; Wilson Cristobal Corrales M. ${ }^{6}$}

1. Docente, M.Sc. Universidad de Granma, Cuba, tlambertg@udg.co.cu.

2. Docente, Universidad de Granma, Cuba, ramonss2018@gmail.com.

3. Docente, Ph. D. Universidad de Granma, Cuba, wceiroc@udg.co.cu.

4. Docente, M.Sc. Universidad Técnica de Cotopaxi, Ecuador, mfernandez@andinanet.net

5. Docente, M.Sc. Universidad Técnica de Cotopaxi, Ecuador, gualomercedeslopez@hotmail.com

6. Docente, Arq. Universidad Técnica de Cotopaxi, Ecuador, wilson.corrales@utc.edu.ec

Citar: Lambert, T., Santiesteban, R., Ceiro, W., Fernández, M., López, G. \& Corrales, W. (2019). Efecto de bioproductos en la producción de Phaseolus vulgaris L. y Arachis hipogea L. Revista de Ciencias Agrícolas. 36(1): 59-66. doi: http://dx.doi.org/10.22267/rcia.193601.98

Recibido: enero 17 de $2018 . \quad$ Aceptado: diciembre 10 de 2018.

\begin{abstract}
RESUMEN
El uso irracional de agroquímicos para aumentar los rendimientos de las plantas, constituye una práctica no amigable con el ambiente. Por esta razón, se realizó una investigación con el objetivo de evaluar el efecto de microorganismos eficientes y quitosano en el crecimiento y rendimiento de Phaseolus vulgaris L. y Arachis hipogea L. Se estableció un experimento en campo en el municipio de Bayamo, provincia Granma - Cuba, entre octubre/2016 y febrero/2017. Se evaluaron efectos de los bioproductos quitosano (Q) y microorganismos eficientes (ME) solos y combinados en el crecimiento y rendimiento de P. vulgaris y A. hipogea. Para ello, se estableció experimento con diseño de Bloques al Azar y cuatro réplicas. El procesamiento estadístico se realizó mediante Análisis de Varianza y la prueba de comparación de Rangos Múltiples de Duncan, $(\mathrm{p} \leq 0,05)$, procesado con STATISTICA, v. 8.0. Los resultados mostraron que la combinación Q+ME potenció el crecimiento de las plantas de maní y frijol, así como el rendimiento.
\end{abstract}


Se evidenciaron los mayores valores de ginóforos/planta, semillas/planta y peso/100 semillas cuando fueron tratados con Q+ME. Así, la combinación quitosano + microorganismos eficientes, surge como una alternativa de biofertilización eficiente en la producción sostenible de frijol y maní.

Palabras clave: Biofertilizantes, microorganismos eficientes, quitosano, producción orgánica.

\begin{abstract}
The agrochemical inadequate use to increase the plants yield, is not an environmentally friendly practice. For this reason, the research objective was to evaluate the effect of efficient-microorganisms and chitosan on growth and yield of Phaseolus vulgaris L and Arachis hipogea L. An experiment was established in field at Bayamo municipality, Granma province in the period October/2016 and February/2017. Chitosan (Q) and efficient-microorganisms (EM) alone and combinations were evaluated on the growth and yield of $P$. vulgaris and A. hipogea. For it, a design of randomized blocks with four replies it was established and an ANOVA and Duncan's test $(p \leq 0.05)$ it was used. The results showed that Q+EM combination potentiated the growth and yield of peanuts and bean plants. With Q+EM were obtained the bigger values of gynophores/plant, seeds/plants and weight/100 seeds. Thus, the chitosan + efficient-microorganism's combination, rise as an biofertilization efficient alternative in the sustainable production of bean and peanuts.
\end{abstract}

Keyword: Biofertilizers, efficient-microorganisms, chitosan, organic production.

\section{INTRODUCCIÓN}

Los cultivos de granos tienen una gran importancia económica en Cuba. En la actualidad el Ministerio de Agricultura (MINAG) trabaja en la sustitución de importaciones de frijol, arroz y maíz. Los cultivos mencionados, así como el maní, constituyen una fuente importante de proteínas, vitaminas y minerales para la alimentación de la población y representan el sustento financiero de muchas familias campesinas (Morales et al., 2016). En Cuba los cultivos de fríjol y maní pese a recibir fertilización convencional, su rendimiento no supera 1,32 y 1,11t.ha-1, respectivamente (Anuario Estadístico de Cuba, 2017; Moreno, 2017).

Sin embargo, se conoce que mediante métodos biotecnológicos se desarrollan alternativas innovadoras para la nutrición de las plantas. En este sentido, se destacan los productos microbianos y orgánicos, que tienen como fin reducir riesgos de contaminación del agroecosistema e incrementar los rendimientos de las plantas
(Chala et al., 2013; Chen et al., 2014; Kuotsu et al., 2014). En tal dirección, muchos agricultores utilizan los microorganismos eficientes (ME), como alternativa a los fertilizantes convencionales (Álvarez et al., 2012). Los ME se consideran como un cultivo mixto de microorganismos benéficos. Estos, al ser inoculados al suelo incrementan la actividad biológica del mismo, así como, en las plantas activan los mecanismos de defensa, potencian la nutrición, el crecimiento y el rendimiento (Núñez et al., 2017).

Los ME contienen microorganismos de tres géneros principales: bacterias, levaduras $\mathrm{y}$ hongos. Las bacterias constituyentes de ME intervienen en la síntesis de aminoácidos, ácidos nucleicos, sustancias bioactivas $\mathrm{y}$ azúcares. Sin embargo, las levaduras se relacionan con la producción de hormonas y enzimas que intervienen en la división celular activa. Sus secreciones son sustratos útiles para la alimentación de otros microorganismos eficientes como los hongos, mientras que los hongos, actúan sobre los componentes de la 
materia orgánica y la degradan, aportando elementos nutritivos a las plantas y sintetizan sustancias bioactivas y antibióticos que regulan las poblaciones de ciertos microorganismos fitopatógenos. Por ello, la acción conjunta de estos grupos microbianos tiene un efecto significativo en la activación de importantes procesos fisiológicos y metabólicos en las plantas (Goswami et al., 2014).

Por otro lado, sustancias orgánicas bioactivas como el Quitosano, extraída fundamentalmente del caparazón de los crustáceos, la cutícula de ciertos insectos y la pared celular de algunas especies fúngicas, posee un amplio uso agrícola. Se utiliza pricipalmente como biofertilizante, movilizador de nutrientes del suelo, activador de mecanismos de defensas de las plantas y estimulador del desarrollo y el rendimiento vegetal (Shehata et al., 2012; Nadège et al., 2015; El-Eleryan, 2015). Por lo tanto, se considera que la acción combinada de ME y Quitosano pueden influir en la nutrición del frijol y el maní, con un consecuente incremento del rendimiento de estas especies vegetales, sin causar afectaciones al ambiente. Teniendo en cuenta lo expuesto, esta investigación se realizó con el objetivo de evaluar el efecto de la aplicación simple y combinada de microorganismos eficientes y quitosano en el crecimiento y el rendimiento de los cultivos de frijol y maní.

\section{MATERIALES Y MÉTODOS}

La investigación se realizó en la Cooperativa de Créditos y Servicios Fortalecida (CCSF) "Anselmo Aldana Medel", ubicada en las coordenadas, Latitud 20.335823 y Longitud -76.635658, municipio Bayamo, provincia Granma, entre octubre del 2016 y febrero del 2017. El suelo donde se establecieron los cultivos fue clasificado como un Fluvisol (Hernández et al., 1999), el cual posee una fertilidad media, $\mathrm{pH}$ ligeramente ácido $(6,5)$, capacidad de intercambio catiónica media, contenido de $\mathrm{P}_{2} \mathrm{O}_{5}$ y $\mathrm{K}_{2} \mathrm{O}$ muy bajo y bajo, respectivamente. Las labores de preparación de suelo de cruce y surca fueron realizadas con el uso de tracción animal. Posteriormente y un día antes a la siembra, se realizó una aplicación de humus de lombriz sólido a una dosis de 4 t.ha $^{-1}$, como mejorador de la fertilidad del suelo.

Se utilizaron dos bioproductos en el experimento Microorganismos Eficientes, LABIOFAM, Granma-Cuba con una concentración microbiana de $5,10 \times 10^{4} ; 26,00 \times 10^{5}$ y $12,11 \times 10^{5} \mathrm{UFC} / \mathrm{mL}$ de bacterias, levaduras y hongos, respectivamente y quitosano (Q), INCA-Cuba con una concentración de $4 \mathrm{~g} / \mathrm{L}$ de i.a. Semillas de $P$. vulgaris var., delicia 364 y A. hipogea var. Cascajal rosado fueron tratadas con cada producto (ME, Q). Para ME la semilla se embebió en una solución al 2\%, mientras que, en el tratamiento Q se embebió en solución al $10 \%$ de i.a. El tiempo de imbibición fue una hora para cada tratamiento. Al tratar la semilla con ambos productos durante una hora, se obtuvo el tratamiento combinado ( $++M E)$. Luego las semillas se sembraron en campo, en parcelas experimentales de $16 \mathrm{~m}^{2}$. Se estableció una semilla/sitio, con distancias de 0,70 m x $0,06 \mathrm{~m}$ y dos semillas/sitio con distancia de 0,60 x 0,10m para maní y fríjol, en su orden. La población por parcelas fue de 380 y 266 plantas de maní y frijol, respectivamente. Las atenciones culturales posterior a la siembra, fueron realizadas de acuerdo con los instructivos técnicos de estos cultivos (MINAG, 2000; MINAG, 2001).

Al presentarse la cosecha se tomaron aleatoriamente 200 plantas por cada parcela, descartando el efecto de borde. En frijol se determinó la altura de las plantas, el número de vainas por planta, las semillas por vaina, el número de semillas por planta, la masa de 100 semillas y el rendimiento. En maní se registró la altura de las plantas, los ginóforos por planta, las semillas por ginóforos, el número de semillas por planta, la masa de 100 semillas y el rendimiento. Se utilizó un diseño de Bloques al Azar con cuatro tratamientos y cuatro réplicas (4x4). Los resultados se evaluaron a través del 
Análisis de Varianza Unifactorial y para aquellas variables que presentaron diferencias estadísticas significativas, se aplicó la prueba de Rangos Múltiples de Duncan $(\mathrm{p} \leq 0,05)$. Se utilizó el paquete STATISTICA versión 8.0 para Windows, en los procedimientos estadísticos.

\section{RESULTADOS Y DISCUSIÓN}

Al analizar la variable altura de las plantas en frijol y maní, se registraron diferencias significativas $(p \leq 0,05)$ entre tratamientos. El mayor crecimiento de frijol se evidenció con Q+ME, comparado con el resto de las variantes experimentales, le prosiguieron $\mathrm{ME}$, Q y finalmente, el control registró el menor crecimiento. En maní, se evidenció que las plantas alcanzaron el mayor crecimiento con el tratamiento $\mathrm{Q}+\mathrm{ME}$, seguido de $\mathrm{ME}$ y $\mathrm{Q}$, que no se diferenciaron entre sí y finalmente, el control registró el menor crecimiento (Tabla 1).

Tabla 1. Efecto de Quitosano y Microorganismos Eficientes en el crecimiento de las plantas de frijol y maní.

\begin{tabular}{ccc}
\hline \multirow{2}{*}{ Tratamientos } & \multicolumn{2}{c}{ Altura de las plantas (cm) } \\
\cline { 2 - 3 } & Frijol & Maní \\
\hline Q+ME & $61,50 \mathrm{a}$ & $38,20 \mathrm{a}$ \\
ME & $59,30 \mathrm{~b}$ & $37,63 \mathrm{~b}$ \\
Q & $58,50 \mathrm{c}$ & $37,20 \mathrm{~b}$ \\
C & $56,30 \mathrm{~d}$ & $36,00 \mathrm{c}$ \\
\hline CV & $\mathbf{2 , 2 7}$ & $\mathbf{2 , 5 4}$ \\
\hline
\end{tabular}

C: control, Q: quitosano, ME: microorganismos eficientes, Q+ME: combinación quitosano+microorganismos eficientes, CV: coeficiente de variación. Letras distintas en las columnas indican diferencias significativas $(\mathrm{p} \leq 0,05)$ según la Prueba de Rangos Múltiples de Duncan.

El resultado expuesto evidenció que la combinación Quitosano y Microorganismos Eficientes registró un 9,23 y 6,11\% de incremento en altura en frijol y maní, respectivamente, comparado con el control. Probablemente, el efecto combinado de los bioproductos activó los procesos metabólicos y fisiológicos en las semillas y con ello, potenció la germinación de las mismas y el consiguiente desarrollo de las plantas. $\mathrm{Al}$ respecto, recientemente en el cultivo de frijol cv. Cuba-Cueto-25 se logró un mayor desarrollo en la longitud de los tallos, el diámetro de los tallos, el número de hojas y el rendimiendo agrícola con la aplicación de Quitosano, comparado en el control (Morales et al., 2016).

Por otro lado, en condiciones de la provincia Las Tunas, Cuba, el maní se desarrolló mejor en los suelos con contenidos medios de materia orgánica y la biofertilización con hongos micorrízicos arbusculares (González y Pupo, 2017), lo cual se relaciona con el resultado obtenido en la presente investigación, donde se evidenció que en un suelo Fluvisol con fertilidad media, se logró el mayor crecimiento del cultivo en la combinación Quitosano y Microorganismos Eficientes. Nadège et al. (2015) en el cultivo de maíz cv. EVDT 97 STR C1 en condiciones de campo, demostraron que la aplicación de Quitosano combinado con rizobacterias y dosis completa de NPK (nitrógeno, fósforo y potasio), incrementó la biomasa vegetal en un $71,43 \%$ comparado con el control.

En plantas de arroz cv. sd20a tratadas con Quitosano se registró entre un 16,57 y 52,00\% de incremento en la altura y la longitud de la raíz, comparado con el control (Molina et al., 2017). Por otra parte, González et al. (2015) evidenciaron que la combinación de Microorganisos Eficientes y Trichoderma harzianum potenció el desarrollo de los sistemas foliar y radical en plántulas de cebolla cv. H-7, lo cual redujo el tiempo de las mismas en el semillero.

$\mathrm{Al}$ analizar las variables componentes del rendimiento en frijol y maní, se encontraron diferencias significativas $(p \leq 0,05)$ entre tratamientos. En frijol, se evidenció que las variables vainas por planta y masa de 100 semillas obtuvieron resultados estadísticos similares, con mayores 
valores en la combinación $\mathrm{Q}+\mathrm{ME}$, comparado con los demás tratamientos. No se diferenciaron entre sí ME y Q, así como, Q y control. Estos últimos alcanzaron los menores valores. Las variables semillas por vaina y semillas por planta, obtuvieron resultados estadísticos similares, registrándose los mayores valores en la combinación $\mathrm{Q}+\mathrm{ME}$, comparado con los demás tratamientos. ME y Q no se diferenciaron entre sí y el control alcanzó los menores valores (Tabla 2).

En maní, la mayor cantidad de ginóforos por planta se obtuvo en la combinación Q+ME. Le siguieron $\mathrm{ME}, \mathrm{Q}$ y finalmente el control registró el menor valor. La mayor cantidad de semillas por ginóforos se registró en los tratamientos Q+ME, ME y $Q$, sin diferencias entre sí y el control registró el menor valor. La mayor cantidad de semillas por planta se constató en la combinación Q+ME comparado con los demás tratamientos. Le siguieron $\mathrm{ME}$ y $\mathrm{Q}$, los cuales no se diferenciaron entre sí y el control evidenció el menor valor. La mayor masa de 100 semillas se alcanzó en la combinación $\mathrm{Q}+\mathrm{ME}$, seguido de ME y $Q$, sin diferencias entre sí, así como, Q y control no se diferenciaron y ambos registraron los menores valores (Tabla 2).

El resultado señala, que donde se aplicaron los bioproductos se experimentó un mejoramiento en las variables componentes del rendimiento. Esto pudo deberse a los efectos que poseen el quitosanoylos microorganismos eficientes sobre la síntesis de sustancias orgánicas estimuladoras del crecimiento como fitohormonas, vitaminas $\mathrm{y}$ metabolitos secundarios que tienen un efecto estimulador del rendimiento y activan mecanismos de defensa de las plantas. Así como, su influencia en una mejor nutrición vegetal (Kearney et al., 2015). Lo que demuestra la aplicabilidad práctica de estos bioproductos en la producción sostenible de frijol y maní.

Al analizar la variable rendimiento en frijol y maní, se observaron diferencias significativas $(p \leq 0,05)$ entre tratamientos. El rendimiento en frijol y maní experimentó un comportamiento estadístico similar, evidenciándose que en ambas especies vegetales el mayor valor se registró en la combinación $\mathrm{Q}+\mathrm{ME}$, le siguieron ME y $Q$, sin diferencias entre sí y finalmente el control registró el menor rendimiento (Figura 1). Los resultados expuestos evidencian un incremento del rendimiento en ambos cultivos en la combinación Q y ME de 61 y 26\%, consecuentemente, comparado con el control. Lo cual señala, que estos bioproductos pudieron tener un efecto beneficioso en la nutrición y la sanidad vegetal, elementos claves para lograr altos rendimientos en condiciones de campo. Los valores de rendimiento alcanzados en la investigación superaron los informados para estas especies, las cuales no rebasan 1 t.ha $^{-1}$ en la provincia Granma (AEC, 2017).

Tabla 2. Efecto de Quitosano y Microorganismos Eficientes sobre los componentes del rendimiento en plantas de frijol y maní.

\begin{tabular}{crrrrrrrr}
\hline & \multicolumn{7}{c}{ Frijol } & Maní \\
\cline { 2 - 8 } Tratamientos & \multicolumn{1}{c}{ VP } & \multicolumn{1}{c}{ SV } & \multicolumn{1}{c}{ SP } & Ms (g) & \multicolumn{1}{c}{ GP } & \multicolumn{1}{c}{ SG } & \multicolumn{1}{c}{ SP } & \multicolumn{1}{c}{ Ms (g) } \\
\hline Q+ME & $10,03 \mathrm{a}$ & $5,90 \mathrm{a}$ & $59,2 \mathrm{a}$ & $18,84 \mathrm{a}$ & $27,9 \mathrm{a}$ & $2,83 \mathrm{a}$ & $80,97 \mathrm{a}$ & $44,37 \mathrm{a}$ \\
ME & $8,93 \mathrm{~b}$ & $5,57 \mathrm{~b}$ & $48,87 \mathrm{~b}$ & $18,27 \mathrm{~b}$ & $26,3 \mathrm{~b}$ & $2,8 \mathrm{a}$ & $72,5 \mathrm{~b}$ & $43,8 \mathrm{~b}$ \\
Q & $8,70 \mathrm{bc}$ & $5,5 \mathrm{~b}$ & $48,87 \mathrm{~b}$ & $17,91 \mathrm{bc}$ & $25,87 \mathrm{c}$ & $2,8 \mathrm{a}$ & $72,13 \mathrm{~b}$ & $43,63 \mathrm{bc}$ \\
$\mathrm{C}$ & $8,60 \mathrm{c}$ & $5,1 \mathrm{c}$ & $43,83 \mathrm{c}$ & $17,54 \mathrm{c}$ & $24,4 \mathrm{~d}$ & $2,1 \mathrm{~b}$ & $65,5 \mathrm{c}$ & $43,3 \mathrm{c}$ \\
CV & $\mathbf{6 , 1 7}$ & $\mathbf{1 1 , 4 5}$ & $\mathbf{1 2 , 7 8}$ & $\mathbf{0 , 8 5}$ & $\mathbf{3 , 0 3}$ & $\mathbf{1 5 , 8 7}$ & $\mathbf{1 3 , 4 1}$ & $\mathbf{1 , 6 3}$ \\
\hline
\end{tabular}

C: Control, Q: Quitosano, ME: Microorganismos Eficientes, Q+ME: Combinación quitosano+microorganismos eficientes, VP: Vainas/planta, SV: Semillas/vaina, SP: Semillas/planta, Ms: Masa de 100 semillas, GP: Ginóforos/planta, SG: Semillas/ ginóforos, CV: Coeficiente de variación. Letras distintas en las columnas indican diferencias significativas $(\mathrm{p} \leq 0,05)$ según la Prueba de Rangos Múltiples de Duncan. 


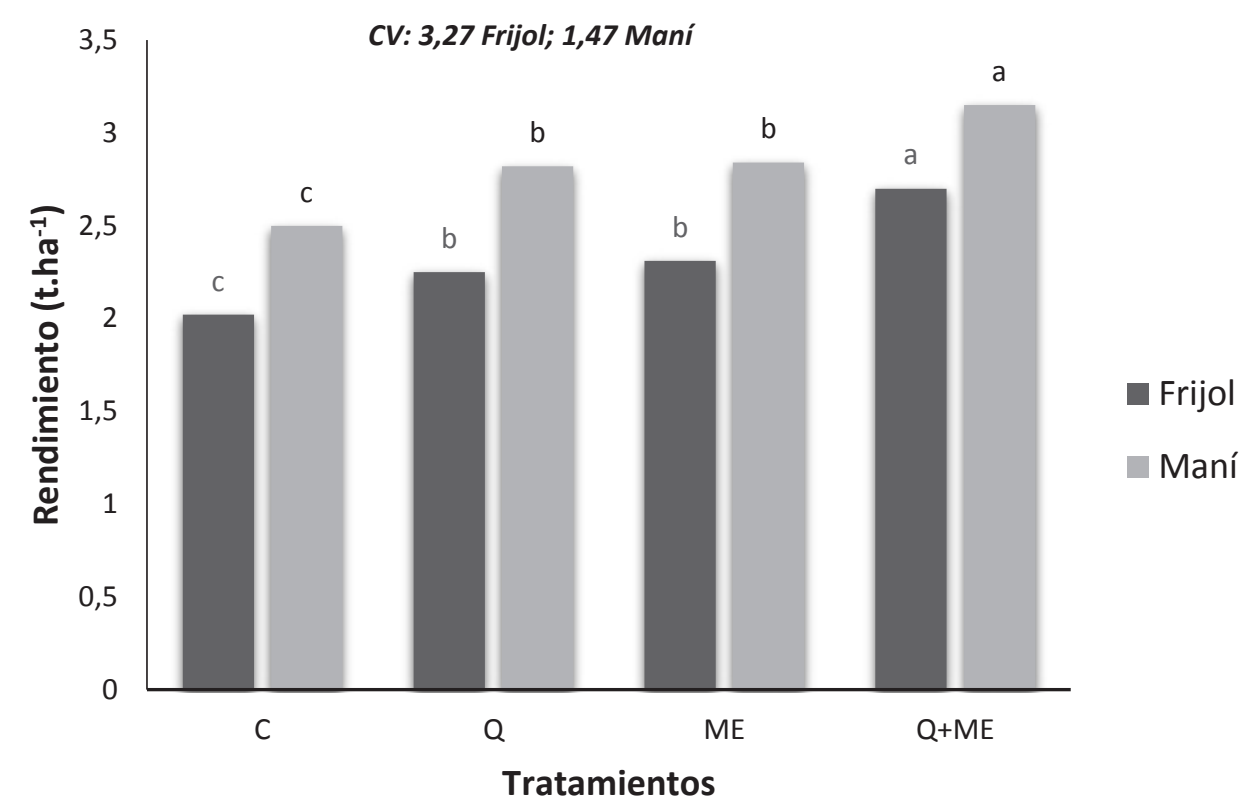

C: Control, Q: Quitosano, ME: Microorganismos Eficientes, Q+ME: Combinación quitosano+microorganismos eficientes, CV: Coeficiente de variación. Letras distintas sobre las barras indican diferencias significativas $(\mathrm{p} \leq 0,05)$ según la prueba de rangos múltiples de Duncan.

Figura 1. Efecto de Quitosano y Microorganismos Eficientes sobre el rendimiento de frijol y maní.

Al respecto, Álvarez et al. (2012) informan que los microorganismos eficientes posibilitan a las plantas la síntesis de aminoácidos, ácidos nucleicos, vitaminas, hormonas y otras sustancias bioactivas que promueven el desarrollo y el rendimiento. Mientras que, el Quitosano tiene un efecto potenciador en la síntesis de sustancias bioactivas, la división y la elongación celular, así como, en la inducción de resistencia en presencia de plagas (Bautista-Baño et al., 2013). Lo expuesto, sugiere que el efecto combinado de estos bioproductos potenció el desarrollo y el rendimiento de las plantas objeto de estudio.

En Cuba, en el año 2016 se establecieron 122 545 ha de frijol, las cuales registraron un rendimiento de 1,32t.ha ${ }^{-1}$ (AEC, 2017). Al comparar dicho rendimiento con el obtenido en esta investigación, se demuestra que la combinación Quitosano y Microorganismos Eficientes experimentó un 104\% de incremento en el rendimiento, lo que evidencia las potencialidades de estos bioproductos en la producción sostenible de frijol. Por otra parte, MINAG (2010) informó que el cultivar de maní Cascajal Rosado puede lograr un potencial de rendimiento de 3,3t.ha-1. No obstante, el promedio histórico en Granma oscila entre $0,8-1,11$ t.ha ${ }^{-1}$ (AEC, 2017). Los resultados obtenidos en este trabajo superaron considerablemente el promedio provincial, por tanto, la aplicación combinada de Microorganismos Eficientes y Quitosano representan una alternativa viable para la producción sostenible de esta especie vegetal.

En plantas de maní, Kearney et al. (2015) demostraron que el uso combinado de tecnologías microbiológicas permite mejorar el rendimiento de esta especie. Mientras que, Díaz et al. (2015) encontraron que la aplicación combinada de hongos y bacterias posibilitó el incremento del rendimiento. Lo expuesto, tiene una estrecha relación con los resultados alcanzados en la presente investigación en la combinación 
Quitosano y Microorganismos Eficientes. Por otro lado, plantas de col de repollo establecidas en organopónico registraron un incremento del rendimiento de $20 \%$ con la aplicación de Microorganismos Eficientes, comparado con el control (Álvarez et al., 2012).

Recientemente, Núñez etal.(2017) demostraron que la zanahoria en condiciones de organopónico incrementó hasta un 19,67\% el rendimiento, con la aplicación de Microorganismos Eficientes, comparado con el control. Mientras que, Navia et al. (2013) informaron que la inoculación de Microorganismos Eficientes al agroecosistema, mejora las propiedades físicas, químicas y biológicas del suelo, así como, el crecimiento, el rendimiento y la calidad de las cosechas, debido principalmente a una mejor nutrición y sanidad de las plantas.

\section{CONCLUSIONES}

Los resultados obtenidos en esta investigación señalan que la aplicación combinada de Quitosano y Microorganismos Eficientes, potencian el desarrollo de las plantas, así como, el rendimiento y sus componentes. Estos bioproductos son una alternativa eficiente para la producción sostenible de $P$. vulgaris y A. hipogea.

Conflicto de intereses: Los autores declaran que no hay conflicto de interés.

\section{REFERENCIAS BIBLIOGRÁFICAS}

AEC- Anuario Estadístico de Cuba. (2017). Agricultura, Ganadería, Silvicultura y Pesca. La Habana, Cuba: Oficina Nacional de Estadísticas e Información (ONEI). 26p.

Álvarez, J., Bárbara, D., González, R.. \& Terence, G. (2012). Evaluación de la aplicación de microorganismos eficientes en col de repollo (Brassica oleracea L.) en condiciones de organopónico semiprotegido. Centro Agrícola. 39(4): 27-30.
Bautista-Baño, S., Sivakumar D., Bello-Pérez A., Villanueva-Arce R. \& Hernández-López, M.A. (2013). Review of the management alternatives for controlling fungi on papaya fruit during the postharvest supply chain. Crop Prot. 49:8-20. doi: 10.1016/j.cropro.2013.02.011

Chala, A., Mohammed, A., Ayalew, A. \& Skinnes, H. (2013). Natural occurrence of aflatoxins in groundnut (Arachis hypogaea L.) from eastern Ethiopia. Food Control. 30 (2): 602-605. doi: https://doi.org/10.1016/j.foodcont.2012.08.023

Chen, M., Li, X., Yang, Q., Chi, X., Pan, L., Chen, N. et al. (2014). Dynamic Succession of Soil Bacterial Community during Continuous Cropping of Peanut (Arachis hypogaea L.). PLoS ONE. 9(7): e101355. doi: https://doi.org/10.1371/journal. pone.0101355

Díaz, Z. M., Kearney, M., Morla, F., Giayetto, O., Barbero, V., Cerion, G. \& Baliña, R. (2015). Boletín de resultados. Ensayos en cultivos de maní, arroz y algodón. Campaña 2013/2014. Recuperado de http://www.monsantobioag.com/global/ las/Products/Documents/Boletines $\% 20 \mathrm{de} \% 20$ Resultados/resultados $\% 20$ en $\% 20$ mani $\% 20$ arroz\%20algodon.pdf

El-Eleryan, E. (2015). Effect of chitosan and green tea on the quality of Washington Navel orange during cold storage. Amer J. Plant Physiol. 10 (1): 43-54. doi: 10.3923/ajpp.2015.43.54

González, G.R. \& Pupo, C.F. (2017). Aplicación de micorrizas: Alternativa ecológica para la disminución o sustitución de fertilizantes químicos en el cultivo del maní. Rev. DELOS: Desarrollo Local Sostenible. 10(29): 3-15.

González, R., Núñez, S,. Hernández, D.B. \& Castro, L.A. (2015). Evaluación de microorganismos eficientes y Trichoderma. Centro Agrícola. 42(2): 25-32.

Goswami, D., Dhandhukia, P., Patel, P. \& Janki, N.T. (2014). Screening of PGPR from saline desert of Kutch: Growth promotion in Arachis hypogea by Bacillus licheniformis A2. Microbiol Res. 169(1): 66-75. doi: https://doi.org/10.1016/j. micres.2013.07.004 
Hernández, A., Pérez J. M., Bosch, D., Rivero, L., Camacho, E. \& Ruiz, J. (1999). Nueva Versión de Clasificación Genética de los Suelos de Cuba. Habana: AGRINFOR. 64p.

Kearney, M., Morla, F., Giayetto, O., Barbero, V. \& Cerioni, G. (2015). Boletín de resultados en el cultivo del maní. Recuperado de http://www. monsantobioag.com/global/las/Products/Documents/Boletines\%20de\%20Resultados/resultados\%20en\%20mani\%20arroz\%20algodon.pdf

Kuotsu, K., Das, A., Anup, L., Munda, R., Ghos, P. \& Ngachan, S. (2014). Land forming and tillage effects on soil properties and productivity of rainfed groundnut (Arachis hypogaea L.) rapeseed (Brassica campestris L.) cropping system in northeastern India. Soil and Till Res. 142:15-24. doi: https://doi.org/10.1016/j.still.2014.04.008

MINAG - Ministerio de la Agricultura. (2000). Instructivo Técnico. Agrotecnia alternativa para el cultivo del frijol (documento inédito). Centro de Enseñanza, Investigación y Transferencia de Tecnología Agropecuaria. Grupo de Granos, Holguín, Cuba. 23p.

MINAG - Ministerio de la Agricultura. (2001). Instructivo Técnico del Maní, Ministerio de la Agricultura, La Habana, Cuba. Recuperado de https:// www.google.com/url?sa=t\&rct=j\&q=\&esrc=s\&s ource $=$ web $\& c d=1 \&$ cad $=$ rja\&uact $=8 \&$ ved $=2$ ahU KEwisnd3r_KfiAhWQwFkKHW9vCL4QFjAAegQI AhAB\&url=http\%3A\%2F\%2Fwww.actaf.co.cu $\%$ 2Fbiblioteca $\% 2$ Fgranos\%2Finstructivo-tecnicoabreviado-del-mani.html\&usg=A0vVaw3urN4d 1KgmYCp1yAPWq-hZ.

MINAG - Ministerio de la Agricultura. (2010). Registro de variedades comerciales de frijol (Phaseolus sp). Ministerio de la Agricultura, La Habana, Cuba. Recuperado de https://www.google.com/ url? sa $=$ t\&rct $=j \& q=\&$ esrc $=$ s\&source $=$ web $\& c d=3 \&$ cad $=$ rja\&uact $=8 \& v e d=2$ ahUKEwiEwLet_KfiAhU Hm1kKHWDCBS8QFjACegQIARAC\&url=http\%3 A\%2F\%2Fwww.actaf.co.cu\%2Frevistas\%2Fagro tecnia_05_2008\%2Fagrot2010-2\%2F18.pdf\&us $\mathrm{g}=$ AOvVaw1VfS6jijTcyQ7DEn7PUsB-6.
Molina, Z., Colina. \& R. (2017). Efecto del uso de quitosano en el mejoramiento del cultivo del arroz (Oryza sativa L. var. sd20a). Rev de Invest Agr y Amb. 8(2): 151-165.

Morales, D., DellAmico, J., Jerez, E., Díaz, Y. \& Martín, R. (2016). Efecto del Quitomax® en el crecimiento y rendimiento del frijol (Phaseolus vulgaris L.). Cultivos Tropicales. 37(1): 142-147.

Moreno, ÁAT.(2017). Uso de abonos orgánicos para el desarrollo sustentable de la escuela Técnica Agronómica Salesiana. Scientific. 2(3): 99-117. doi: https://doi.org/10.29394/ scientific.issn.2542-2987.2017.2.3.5.99-117

Nadège, A., Agbodjato, P., Noumavo, A., Adjanohoun, G., Dagbenonbakin, M., Falcón, R., Noval, P. B. \& Lamine, B.M. (2015). Response of maize (Zea mays L.) crop to biofertilization with plant growth promoting rhizobacteria and chitosan under field conditions. J. Exp Biol Agric Sci. 3(6): 567-574.

Navia, C., Zemanate, C., Morales, V., Prado, F. \& Albán, L. (2013). Evaluación de diferentes formulaciones de compostaje a partir de residuos de cosecha de tomate. Rev Biotec Sec Agrop Agroind. 2: 165-173.

Núñez, S., Liriano, D.B., Pérez, H., Placeres, E. \& Sianeh, Z. (2017). Respuesta de Daucus carota, L. a la aplicación de microorganismos nativos en condiciones de organopónico. Centro Agrícola. 44(2): 29-35.

Shehata, S.A., Fawzy, Z. F. \& El-Ramady, H.R. (2012). Response of cucumber plants to foliar application of chitosan and yeast under greenhouse conditions. Aust J. Bas App Sci. 6(4): 63-71. doi: https://doi.org/10.1134/ S0003683816050124 